\title{
Representational Organization of Novel Task Sets during Proactive Encoding
}

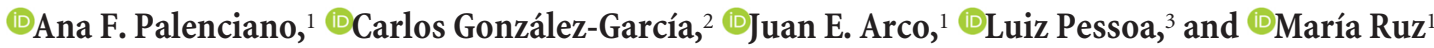 \\ ${ }^{1}$ Mind, Brain, and Behavior Research Center, University of Granada, 18011, Granada, Spain, ${ }^{2}$ Department of Experimental Psychology, Ghent University, \\ 9000, Ghent, Belgium, and ${ }^{3}$ Psychology Department, University of Maryland 20742
}

Recent multivariate analyses of brain data have boosted our understanding of the organizational principles that shape neural coding. However, most of this progress has focused on perceptual visual regions (Connolly et al., 2012), whereas far less is known about the organization of more abstract, action-oriented representations. In this study, we focused on humans' remarkable ability to turn novel instructions into actions. While previous research shows that instruction encoding is tightly linked to proactive activations in frontoparietal brain regions, little is known about the structure that orchestrates such anticipatory representation. We collected fMRI data while participants (both males and females) followed novel complex verbal rules that varied across control-related variables (integrating within/across stimuli dimensions, response complexity, target category) and reward expectations. Using representational similarity analysis (Kriegeskorte et al., 2008), we explored where in the brain these variables explained the organization of novel task encoding, and whether motivation modulated these representational spaces. Instruction representations in the lateral PFC were structured by the three control-related variables, whereas intraparietal sulcus encoded response complexity and the fusiform gyrus and precuneus organized its activity according to the relevant stimulus category. Reward exerted a general effect, increasing the representational similarity among different instructions, which was robustly correlated with behavioral improvements. Overall, our results highlight the flexibility of proactive task encoding, governed by distinct representational organizations in specific brain regions. They also stress the variability of motivation-control interactions, which appear to be highly dependent on task attributes, such as complexity or novelty.

Key words: fMRI; frontoparietal network; instructed-behavior; motivation; proactive control; representational similarity analysis

\section{Significance Statement}

In comparison with other primates, humans display a remarkable success in novel task contexts thanks to our ability to transform instructions into effective actions. This skill is associated with proactive task-set reconfigurations in frontoparietal cortices. It remains yet unknown, however, how the brain encodes in anticipation the flexible, rich repertoire of novel tasks that we can achieve. Here we explored cognitive control and motivation-related variables that might orchestrate the representational space for novel instructions. Our results showed that different dimensions become relevant for task prospective encoding, depending on the brain region, and that the lateral PFC simultaneously organized task representations following different control-related variables. Motivation exerted a general modulation upon this process, diminishing rather than increasing distances among instruction representations.

\section{Introduction}

Humans quickly learn from instructions which elements are relevant in a context and their respective appropriate actions. These

Received April 1, 2019; revised July 19, 2019; accepted Aug. 13, 2019.

Author contributions: A.F.P., C.G.-G., L.P., and M.R. designed research; A.F.P. performed research; A.F.P. and J.E.A. analyzed data; A.F.P. wrote the first draft of the paper; A.F.P., C.G.-G., L.P., and M.R. edited the paper; A.F.P. and M.R. wrote the paper.

This work was supported by the Spanish Ministry of Science and Innovation (PSI2016-78236-P) and the Spanish Education, Culture and Sports Ministry (FPU2014/04271 and EST16/00772 to A.F.P.). This research is part of A.F.P.'s activities for the Psychology Graduate Program of the University of Granada. We thank Srikanth Padmala for valuable help during the planning and implementation of the different fMRI data analysis used in the current experiment. parameters are encoded proactively in our brain in an actionbased code (Brass et al., 2017; Cole et al., 2017), preparing our perceptual and motor systems in advance (Cole et al., 2013) and facilitating success in novel environments. Instructed behavior is thus critical to avoid less effective and slow trial-and-error learning, and also enables the social transmission of task procedures. There is scarce knowledge, however, about how the informa-

The authors declare no competing financial interests. Correspondence should be addressed to María Ruz at mruz@ugr.es. https://doi.org/10.1523/JNEUROSCI.0725-19.2019 Copyright $\odot 2019$ the authors 
tional and motivational content of novel instructions organizes neural activity in a proactive manner.

Behavioral results support the role of proactive control (Braver, 2012) on instructed action (Luria, 1966; Duncan et al., 2008; e.g., Liefooghe et al., 2012; see also Cole et al., 2018). Recently, neuroimaging studies have revealed a link between novel instruction preparation and the frontoparietal (FP) network (e.g., Cole et al., 2010; Hartstra et al., 2011; Palenciano et al., 2019). The middle (MFG) and inferior (IFG) frontal gyri, and the inferior frontal sulcus (IFS), together with the intraparietal sulcus (IPS), encode novel instruction content both in multivoxel activity patterns (González-García et al., 2017; Muhle-Karbe et al., 2017; Bourguignon et al., 2018) and distributed functional connectivity (Cole et al., 2013). Crucially, the fidelity of information encoding is linked to the intention to implement the instruction (vs mere memorization demands; Muhle-Karbe et al., 2017; Bourguignon et al., 2018), and it is also closely related to the efficiency of behavior (Cole et al., 2016; González-García et al., 2017). Nonetheless, while current studies have mainly focused on decoding the upcoming target category (González-García et al., 2017; Muhle-Karbe et al., 2017), the wider organizational structure that shapes anticipatory task representation remains unknown. To study the relevant dimensions organizing novel instruction encoding, we selected three variables known to be relevant for proactive control.

Task preparation consists of a two-step process (Rubinstein et al., 2001), composed first by an abstract goal reconfiguration and second by the activation of specific stimulus-response contingencies (De Baene and Brass, 2014; Muhle-Karbe et al., 2014). Our study exploited these two phases. First, in relation to the high-level task goal setting, we manipulated the integration of information within or across feature dimensions of stimuli (Rigotti et al., 2013), a variable traditionally linked to task complexity and top-down attention (e.g., Treisman and Gelade, 1980). Second, the stimulus-response reconfiguration process was manipulated by the response set complexity, requiring single or sequential motor responses. Moreover, to explore stimuli-specific preparatory mechanisms previously documented (Sakai and Passingham, 2003, 2006; e.g., González-García et al., 2016), we also manipulated the relevant target category.

Finally, cognitive control and motivation maintain an intricate relationship during task preparation (Pessoa, 2009, 2017). Reward expectation boosts cue-locked activity across the FP network (Parro et al., 2018), and it has been recently linked to stronger anticipatory rule encoding (Etzel et al., 2016). Nonetheless, contradictory findings have also been observed (Wisniewski et al., 2019), and a comprehensive characterization of this interaction in complex, novel scenarios is still pending. Consequently, we included economic incentives in our paradigm and assessed the nature of their effect on instruction preparation. By varying these four variables (dimension integration, response-set complexity, target category, and reward), we built a set of novel, verbal instructions that were followed by healthy participants while fMRI data were collected. Using representation similarity analysis (RSA; Kriegeskorte et al., 2008), we assessed the extent to which each of our control-related variables organized instruction encoding, as well as the effect of motivation upon this organization.

\section{Materials and Methods}

\section{Participants}

Thirty-six students from the University of Granada completed the experimental paradigm inside an MRI scanner ( 16 women, mean age $=22.97$ years, $\mathrm{SD}=3.32$ years). All of them were right-handed, with normal or corrected-to-normal vision, and native Spanish speakers. In exchange for their participation, they received between 20 and $40 €$, depending on their performance on the rewarded trials (see below). They all signed a consent form approved by the Ethics Committee of the University of Granada. Four participants were later excluded due to excess of head movement ( $>3 \mathrm{~mm}$ ) or poor performance ( $<70 \%$ of correct responses).

\section{Apparatus, stimuli, and procedure}

For the experiment, we built a set of 192 different novel verbal instructions. Each instruction referred to two independent conditions about faces or food items that could be met or not by the upcoming grids, and their associated responses (e.g.: "If there are two women and an additional sad person, press A; if not, press $L$ "). The conditions in the instructions referred to several dimensions of the stimuli: gender (woman, man), race (black, white), emotion (happy, sad) and size (big, small) of faces, or kind (fruit, vegetable), color (green, yellow), form (round, elongated), and size (big, small) of food items.

Instructions were created by manipulating in an orthogonal manner: (1) the integration of stimuli dimensions (within vs across dimensions), (2) the response set required (single vs sequential), and (3) the category of the relevant stimuli that they referred to (faces vs food). For example, the instruction "If there is a woman and there is a man, press A; if not, press $L$ " involves within-dimension integration (i.e., gender), requires a single response (a left " $A$ " or a right " $L$ " index button press) and is face-related. On the other hand, "If there is a fruit and a small food item, press AL; if not, press $L A$ " requires across-dimension integration (the type of food and its size), demands a sequence of two button presses to respond, and is foodrelated. Instructions referred to 2, 3, or 4 stimuli of the target grid. Equivalent trials were created for the different levels of these three variables.

In addition, we included motivation as another variable: half of the instructions were associated with the possibility of receiving an economic reward if responses were fast and accurate, whereas the other half were nonrewarded. To do so, we split our 192 instructions into two equivalent sets in terms of the manipulations of the other independent variables, and also regarding the specific attributes specified (e.g., the same number of instructions referring to happy faces in both groups). We counterbalanced across participants the assignment of these two halves to the rewarded and nonrewarded conditions. The reward status of each trial was indicated by a cue consisting of either a plus $(+)$ or a cross $(x)$ sign, in either silhouette or filled in black. We counterbalanced across participants whether they should attend to the shape (plus vs cross) or the appearance (contour vs filled sign) to obtain the reward information. This way, each participant had two different cues indicating each motivation condition, preventing a one-to-one mapping between reward expectation and visual cue identity, which otherwise could generate spurious confounds in further analysis.

For each instruction, we created two grids of stimuli: one that fulfilled the conditions instructed and another one that did not. We counterbalanced them so that individual participants saw only one of the two instruction-grid pairings. All grids were unique combinations of images of 4 faces and 4 food items, which were pseudo-randomly selected from a pool of 32 pictures, composed of 16 faces pictures ( 8 different identities, half of them women and half men, half with happy expressions and half with sad ones, half white and half black, appearing each of them in large and small sizes), extracted from the NimStim database (Tottenham et al., 2009), and 16 food pictures ( 8 different items, half of them vegetables and half fruits, half in green color and half in yellow, half with a round shape and half elongated, appearing each of them in large and small sizes) obtained from available sources on the internet (all of them with Creative Commons license). Upon target presentation, the responses required were always one or two sequential button presses, performed with the left (" $A$ ") and/or right ("L") index. The sequence of trial events is depicted in Figure 1. Each trial started with a jittered fixation point $\left(0.5^{\circ}\right)$, with a duration that ranged from 4500 to $7500 \mathrm{~ms}$, in steps of $500 \mathrm{~ms}$ (mean = $5750 \mathrm{~ms})$. Then, a reward cue was presented $\left(1.5^{\circ} ; 2000 \mathrm{~ms}\right)$, followed by the instruction $\left(25.75^{\circ} ; 2500 \mathrm{~ms}\right)$. Next, a second jittered fixation appeared (with the same characteristics as the previous one), and the target $\operatorname{grid}\left(21^{\circ}\right)$ was presented for $2500 \mathrm{~ms}$, where participants were required to respond. Afterward, a feedback symbol was presented $\left(1.65^{\circ} ; 500 \mathrm{~ms}\right)$, 
indicating whether the participant had earned money in that trial (with a Euro symbol), whether the response was correct but no money was achieved (tick symbol), or whether the response was incorrect (cross symbol).

Before being scanned, participants completed a behavioral practice session. They received indications about how to perform the task, as well as details on how rewards would be administered, emphasizing that both accurate and fast responses were needed to accumulate money for a maximum of $40 €$. Specifically, they were informed that they would receive $20 €$ for their time and that the rest of the compensation would depend on their performance on rewarded trials: the initial extra increases would be easier to earn while approaching the upper limit of the payment would require a higher accuracy rate. Then, they performed a simple discrimination task with the different reward cues; and after that, they practiced the instruction-following task, completing one block of 32 trials. Practice instructions were drawn from a separate set (which was equivalent in all the parameters specified above) and were not used in the MRI experiment to maintain trial novelty. Participants repeated the practice block as many times as needed to obtain an accuracy rate $>75 \%$ (on average, participants performed the practice block 1.75 times). Once this phase was completed, the experimental paradigm was performed inside the scanner. This was composed by the full 192 instructions set, presented in six different runs ( 32 trials each). All runs included an equal number of face and food-related, single and sequential responses, within and across-dimension integration and rewarded and nonrewarded instructions. Overall, participants spent $\sim 90$ min inside the MRI scanner.

\section{Experimental design and behavioral statistical analysis}

Our task was built following a 4-way factorial design, in which the following within-subjects independent variables were orthogonally manipulated: (1) dimension integration, (2) response set complexity, (3) target category, and (4) reward.

We conducted an a priori power analysis to compute sample size. Using the PANGEA software (https://jakewestfall.shinyapps.io/pangea/), we calculated the minimum number of participants to detect a behavioral twoway interaction term (i.e., between reward and any other proactive control-related variable), assuming a medium effect size (Cohen's $d=0.3$ ).

We used SPSS Statistics version 20 software (IBM) to analyze accuracy and reaction time (RT) data. We conducted two repeated-measures ANOVAs, specifying four factors corresponding to our independent variables. To explore significant interaction terms, we performed further post hoc tests, using a Bonferroni correction for multiple comparisons.

\section{fMRI preprocessing}

MRI data were acquired using a 3 tesla Siemens Trio scanner located at the Mind, Brain, and Behavior Research Center (University of Granada, Spain). Functional images were collected using a T2* EPI sequence $\left(\mathrm{TR}=2210 \mathrm{~ms}, \mathrm{TE}=23 \mathrm{~ms}\right.$, flip angle $\left.=70^{\circ}\right)$. Each volume consisted of 40 slices, obtained in descending order, with $2.3 \mathrm{~mm}$ of thickness (gap = $20 \%$, voxel size $=3 \mathrm{~mm}^{3}$ ). A total of 1716 volumes were obtained, in 6 runs of 286 volumes each. We also acquired a high-resolution anatomical T1-weighted image (192 slices of $1 \mathrm{~mm}, \mathrm{TR}=2500 \mathrm{~ms}, \mathrm{TE}=3.69 \mathrm{~ms}$, flip angle $=7^{\circ}$, voxel size $=1 \mathrm{~mm}^{3}$ ).

The functional images were preprocessed and analyzed with SPM12 (http://www.fil.ion.ucl.ac.uk/spm/software/spm12/), with the exception of single-trial parameter estimation (see RSA), which was conducted on AFNI. After discarding the first four volumes of each run to allow for stabilization of the signal, the images were spatially realigned and slice-time corrected. Then, the participants' structural T1 image, which had been

Figure 1. Sequence of events in a single trial. ISI, Interstimulus interval.

coregistered with the EPI volumes, was segmented to obtain the transformation matrices needed to normalize the functional images to the MNI space. Finally, they were smoothed with an $8 \mathrm{~mm}$ FWHM Gaussian kernel. The full preprocessing pipeline was completed before conducting the univariate analysis, whereas only realigned and slice-timing corrected images were used for the multivariate tests (see fMRI statistical analysis). In the latter, normalization and smoothing were performed after the individual-level analysis, following the same strategy as above.

\section{fMRI statistical analysis}

Control univariate analysis. We first conducted a univariate standard GLM, modeling each of the 16 combinations of our variables (e.g., within-dimension integration/simple response required/faces-related/ rewarded) and specifying two regressors per trial: one for the encoding phase (from the reward cue until the end of the instruction) and another for the implementation stage (encompassing the target grid presentation and until the end of the feedback cue). All regressors were convolved with the canonical hemodynamic response function. We also added error trials and six motion parameters as nuisance regressors, and a high-pass filter of $128 \mathrm{~s}$ to avoid low-frequency noise.

The rationale of this analysis was to check the effect of motivation during the encoding of novel instructions with the aim of ensuring that our manipulation successfully generated typical reward-related patterns of activation (Parro et al., 2018). This was done by performing $t$ tests at the individual (first) level, contrasting rewarded versus nonrewarded encoding regressors, and carrying these statistical maps to a group onesample $t$ test. The result was clusterwise FWE-corrected for multiple comparison at $p<0.05$ (from an initial threshold of $p<0.001$ and $k=10$ ). With this approach, we obtained one large cluster that extended across multiple brain regions. To obtain smaller, anatomically coherent clusters, we used a stricter threshold (uncorrected cluster-forming threshold of $p<0.0001$, with the corresponding FWE correction at $p<0.05$ ), as done previously (e.g., Dumontheil et al., 2011; Palenciano et al., 2019).

\section{$R S A$}

We conducted a series of multivariate RSAs, following a two-step approach. First, we analyzed whole-brain data, using a searchlight approach, to find regions encoding novel instructions according to each of our three control-related variables. Second, we used the significant areas as ROIs and focused on them to explore the effect of reward on their representational geometry.

Whole-brain model-based RSA. We first studied whether the representational structure of novel instructions was explained by three variables 
a

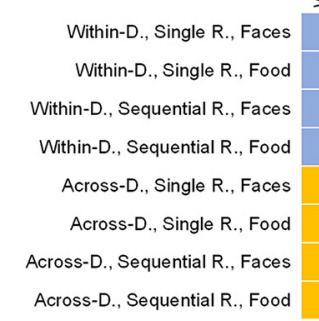

Across-D., Sequential R., Food

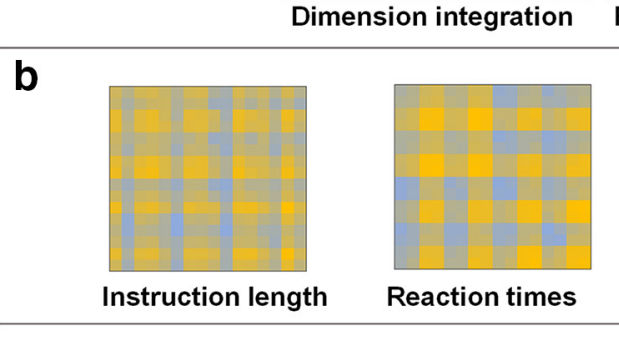

d

e.g.: Target category mode

R+ trials

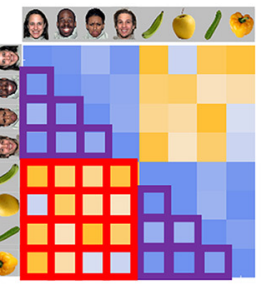

Hypothesis 1:

$\left(\right.$ Same Cond $\mathrm{R}_{+}-$Different Cond $\left._{\mathrm{R}_{+}}\right)>$

(Same Cond $_{\mathrm{NR}}$ - Different Cond $\mathrm{NR}$ )

Hypothesis 2:

$\left(\right.$ Same Cond $\mathrm{R}_{+}+$Different Cond $\left._{\mathrm{R}_{+}}\right)>$

(Same Cond $_{\mathrm{NR}}+$ Different Cond $\mathrm{NR}_{\mathrm{N}}$ )

\section{Theoretical DSMs}

\begin{tabular}{|lc|}
\hline \multicolumn{2}{|c|}{ Dissimilarity } \\
\hline $\min$ & $\max$ \\
\hline
\end{tabular}

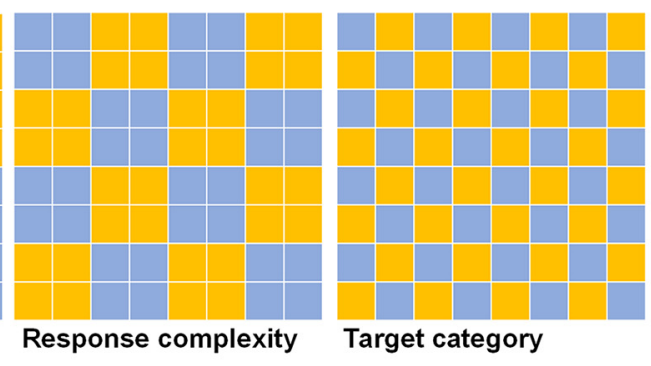

C

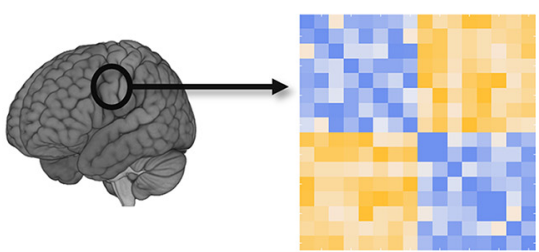

Neural DSM
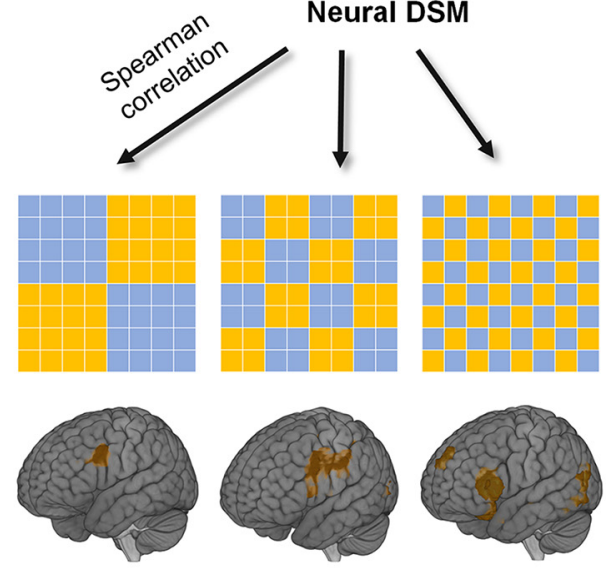

Figure 2. Main analysis procedure. $\boldsymbol{a}$, Theoretical RDMs used in the RSA. Within/Across-D, Within-dimension and across-dimension integration; Single/Sequential R, single response and sequential response. $\boldsymbol{b}$, RDMs capturing differences in instruction length (number of letters) and RT, included in a multiple regression analysis together with matrices shown in $\boldsymbol{a}$ to control for the effect of these two variables. $c$, Following a searchlight approach, we extracted the neural RDM at each brain location and compared it (via Spearman correlation) with our three theoretical RDMs. As a result, we obtained three whole-brain correlation maps, one per model. $\boldsymbol{d}$, To assess the effect of motivation, for each region, significant in $\boldsymbol{c}$, we extracted the neural RDMs from rewarded ( $R+$ ) and nonrewarded (NR) trials. To study potential interactions of reward expectation and the corresponding model variable (Hypothesis 1), we averaged the dissimilarity values among same-condition and different-condition trials and tested whether the subtraction among these two values was higher in the rewarded condition (using Wilcoxon signed-rank test). We also checked for a general increase in dissimilarities associated with reward (Hypothesis 2). All matrices in the figure were simplified for visualization purposes by averaging cells within conditions. $\boldsymbol{b}$, The matrices were further averaged across the sample. $\boldsymbol{d}$, Matrices display only one task variable (collapsing between the remaining two) to highlight the analysis logic. In all the analyses, however, trialwise and single subject matrices were used.

related to cognitive control preparation: dimension integration, response set complexity, and target category. Importantly, we specifically wanted to explore this during the initial encoding stage, where proactive task-set reconfiguration takes place. To do so, we first obtained trial-bytrial estimations of our signal, following a least-square-sum approach (Turner, 2010) to ensure the smallest possible collinearity among regressors (Arco et al., 2018). We generated and estimated one separate model per trial, in which we defined: (1) a regressor isolating the encoding phase of the individual trial of interest; (2) a second regressor containing the rest of trials (encoding phase) of the same condition; (3) 31 additional regressors encompassing the rest of conditions at the encoding and im- plementation phases (as in the GLM specified above); and (4) nuisance regressors (movement, errors). To do so, we used AFNI's function 3dLSS (https://afni.nimh.nih.gov/pub/dist/doc/program_help/3dLSS.html). Once the trialwise parameter images were obtained, the rest of the RSA was performed with the Decoding Toolbox (Hebart et al., 2014).

In our analysis, we compared three theoretical models of representational organization (one per preparation-related independent variable) with the empirical one, built from spatially distributed activity patterns. To do so, we used a spherical searchlight (radius: 4 voxels) and applied it to the whole brain (Kriegeskorte et al., 2006). First, we built three theoretical representational dissimilarity matrices (RDMs) (Fig. 2a), which 
captured the expected dissimilarity (represented with 0 and $1 \mathrm{~s}$ ) between pairs of trials, according to the corresponding variables of interest. For example, in the Category RDM, dissimilarity is expected to be minimal within pairs of trials that refer either to faces or to food, whereas it should be maximal between pairs of trials that refer to different target categories. Then, in each iteration of the searchlight, we generated a neural RDM, using a measure of distance based on Pearson correlation. Specifically, we extracted the corresponding single-trial $\beta$ values of the voxels involved, correlated each pair of the trials' activity patterns, and subtracted that value from 1. Afterward, this neural RDM was Spearman-correlated with the theoretical ones (Fig. 2c), and the coefficients were normalized with Fisher's $z$ transformation and assigned to the central voxel of the searchlight sphere. Importantly, both theoretical and neural matrices were built trialwise (i.e., not averaging within conditions) and, thus, were fully symmetrical with a diagonal of $0 \mathrm{~s}$. Consequently, only the lower triangle of the matrices, excluding the diagonal, was included in the correlation to avoid inflated positive results (Ritchie et al., 2017). After iterating the searchlight across the whole brain, we obtained three maps per participant, representing how well the representational geometry in different regions matched the one expected by each of our three theoretical models.

Statistical significance was assessed nonparametrically via permutation testing, as proposed by Stelzer et al. (2013). We first performed 100 permutations at the individual level, where trial labels were randomly shifted and the whole analysis was repeated. Then, at the group level, we resampled 50,000 times one of the permuted maps of each subject and averaged them. The resulting bootstrapped group maps were used to build a voxelwise null distribution of correlation values, which was used to extract the correlation coefficient coinciding with a probability of 0.001 of the right-tailed area of the distribution (i.e., linked to a $p<$ 0.001 ) of each individual voxel. The group map of the results was then thresholded using these values. From the bootstrapped maps, we also built a null distribution of cluster sizes (Stelzer et al., 2013), which determined the probability of each cluster extent under the null distribution. We used this to assign the corresponding $p$ value to the surviving clusters of the group results map, and FWE-corrected $(p<0.05)$ them to control for multiple comparisons.

We performed a further conjunction test to find areas sharing the three representational organization schemes. To do so, we thresholded ( $p<$ 0.05 , FWE-corrected) and binarized the three maps from the previous step, and obtained the overlapping voxels (Nichols et al., 2005).

Importantly, the RSA results could be influenced by other variables statistically related to our manipulations (Popov et al., 2018), such as instructions' length and speed of responses, which differed slightly between conditions. To examine their influence on the results, we performed an additional multiple regression analysis taking both variables into account. We built two different RDMs (Fig. 2b) in which each cell contained the absolute difference in the number of letters (instruction's length RDM) or RT (response speed RDM), respectively, between specific pairs of instructions. We then used them as regressors together with the three proactive control-related RDMs, predicting the neural pattern of dissimilarities in each iteration of a searchlight. The regressors were built vectorizing the lower triangle of the RDM, excluding the diagonal values. It is important to note that there were small, but still significant, correlations among some of the regressors included in the analysis. Specifically, dimension integration correlated with instruction length and RT, and target category did so with instruction length. To assess the impact of these correlations on the regression estimation, we computed variance inflation factors (Mumford et al., 2015), an index of the regressors' collinearity. For our five models, and in all the participants, variance inflation factors were always $<1.1$ (being 5 a typical cutoff above which the estimation would be compromised; Mumford et al., 2015). Thus, even despite the relationship among variables, the results of our main analyses are still meaningful. The corresponding $\beta$ weight maps obtained showed the regions where the effect of our variables of interest remained significant, even when instruction's length and response speed were included.

Finally, even when the distance measure used to build the neural RDMs (i.e., Pearson correlation) is insensitive to differences in mean signal intensity between conditions, differences in signal variance could be affecting it (Walther et al., 2016). For that reason, these analyses as well as the reward-related tests (see below) were repeated after a $z$ normalization of the multivoxel activity patterns, ensuring equal mean $(0)$ and SD (1) across all pairs of trials. The results thus obtained did not differ from the initial non-normalized ones, so we do not report them here.

ROI-based RSA. The previous analysis identified brain areas encoding instructions according to each one of three proactive control variables separately. We next ran ROI analyses to further explore the role of the three variables for task coding in these regions. Specifically, we estimated the extent to which each of the manipulated control variables explained the neural organization in the ROIs identified in the previous analysis. We followed a leave-one-subject-out (LOSO) cross-validation procedure (Esterman et al., 2010), using the searchlight maps obtained before. First, we identified regions sensitive to each of the three models for each participant, running a group-level $t$ test with the corresponding maps from the rest of the sample (i.e., excluding their own data). Significant clusters showing consistency across all LOSO iterations were selected as ROIs, and inverse normalized to the participants' native space. In a second step, we estimated the ROI RDMs and correlated them with the three theoretical models. Importantly, thanks to the LOSO procedure, we avoided circularity in the analysis, as independent data were used to select the ROIs and to compute correlations with the models. The correlation coefficients (for each participant, one per ROI and model) were then introduced into a repeated-measures ANOVA, with ROI and model as factors, and the interaction term was examined to detect heterogeneity in task encoding organization across regions (Reverberi et al., 2012). Interactions were further characterized by one-sample $t$ tests, to determine which models had an effect on the different regions studied. Whenever the normality assumption was not met (assessed with the Shapiro-Wilk test), we used Wilcoxon signed-rank tests instead. All $p$ values were Bonferroni-corrected for multiple comparisons, adjusting them to the number of ROIs explored.

Additionally, we aimed to extrapolate our findings to regions consistently found in the literature during both practiced (e.g., Woolgar et al., 2011) and novel (e.g., González-García et al., 2017) task preparation, and in general, when demanding cognitive processing is deployed (Duncan, 2010). This set of brain areas belong to the multiple demand network (Duncan, 2010), which includes the bilateral rostrolateral prefrontal cortex (RLPFC), MFG, IFS, anterior insula/frontal operculum (aIfO) area, IPS, anterior cingulate cortex (ACC), and presupplementary motor area (pre-SMA). To assess the organization of novel task encoding across this multiple demand network, we used functionally derived masks of its nodes (Fedorenko et al., 2013; template available at http://imaging.mrccbu.cam.ac.uk/imaging/MDsystem), inverse normalized them to the participants' native space, and followed the same ROI approach as above, extracting each ROI RDM and correlating it with the models' matrices. Again, correlation coefficients were entered into a repeated-measures ANOVA with ROI and model as factors, interactions were examined, and finally, a series of one-sample $t$ tests (or Wilcoxon signed-rank test when normality was violated) were conducted.

Analysis of reward-related effects on RSA results. A final goal of our study was to assess whether the representational space of novel instructions was affected by motivation. Our initial hypothesis was that reward would polarize the representational geometry, enhancing the effect of our control-related variables at structuring rule encoding. In other words, and taking as an example the target category variable, we assessed whether reward expectations would increase the distance between representations of instructions referring to different stimulus categories (in extension to the other variables, indicated as different-condition dissimilarity) while decreasing the distance among those referring to same target category (same-condition dissimilarity). Our second, alternative hypothesis was that reward would exert a general effect, globally increasing the distances among instruction representations, independently of the other variables manipulated. In this sense, we expected that both different and samecondition dissimilarity would be increased in rewarded trials, in comparison with nonrewarded ones. The two possibilities would be compatible with previous findings showing that reward expectancy enhances rule decodability (Etzel et al., 2016). 

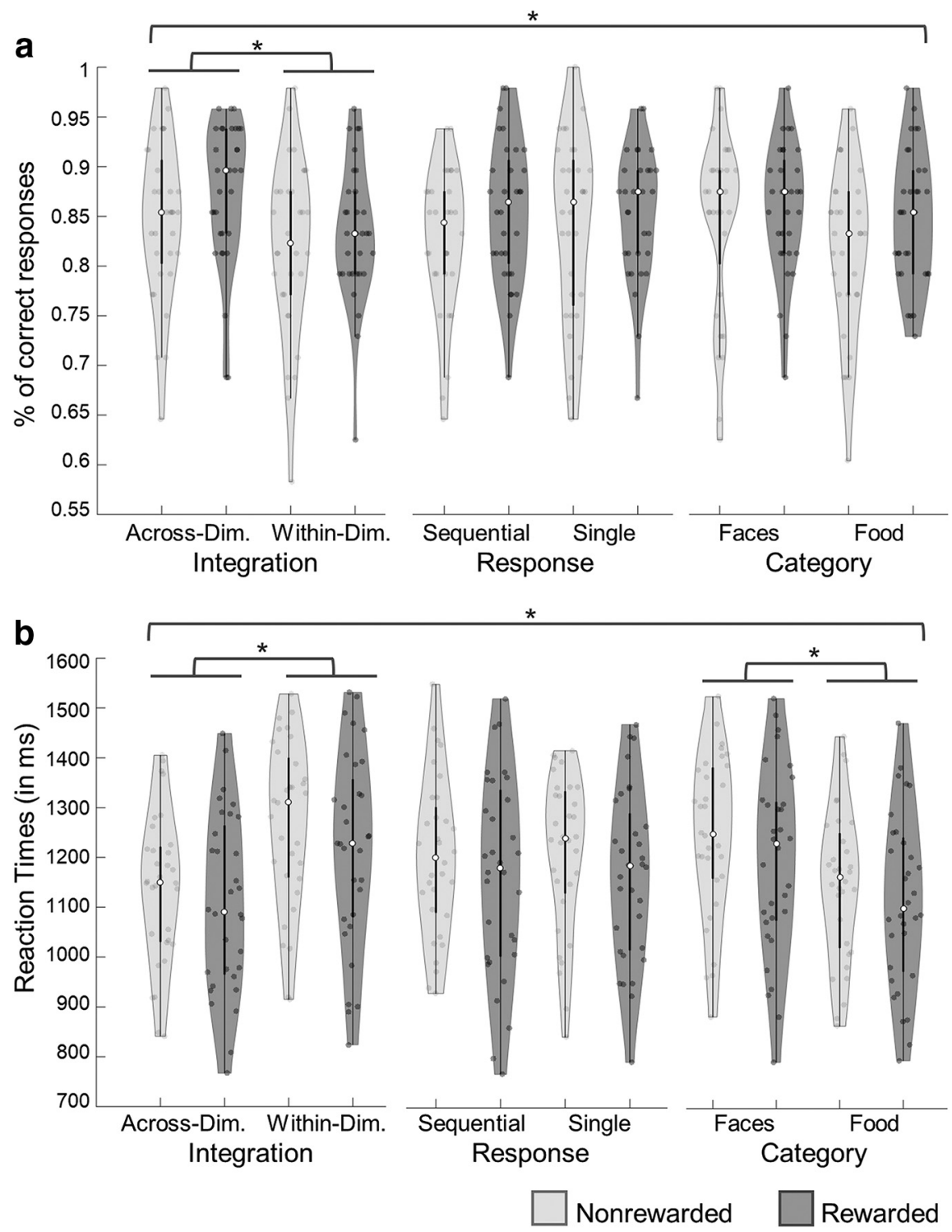

Figure 3. Behavioral data. Violin plots showing correct responses $(\boldsymbol{a})$ and RT $(\boldsymbol{b})$ data for each condition, in rewarded and nonrewarded trials. Asterisks indicate $p<0.05$ for main effects in the repeated-measures ANOVA.

To test these two hypotheses, we run ROI analyses (Fig. $2 d$ ) for each of our control-related variables, focusing on the regions that resulted statistically significant in the main RSA. To do so, at the individual level and for each variable, we first ran a searchlight and generated four wholebrain maps containing dissimilarity values among: (1) same-condition rewarded trials; (2) different-conditions rewarded trials; (3) samecondition nonrewarded trials; and (4) different-conditions nonrewarded trials. These values were the result of averaging and normalizing (with the Fisher transformation) the pertinent cells of the neural RDM (see, e.g., Fig. 2d) in each searchlight iteration. The maps thus obtained were normalized to the MNI space, so we could extract participants' mean dissimilarities for each of our ROIs using MarsBar (Brett et al., 2002). After that, and for each ROI and variable, we conducted two Wilcoxon signed-rank tests (Nili et al., 2014). First, to assess our main hypothesis, we tested whether (DifferentCond. . $_{\text {Rewarded }}-$ SameCond. $_{\text {Rewarded }}$ ) $>$ (DifferentCond. NonRewarded $^{-}$SameCond. NonRewarded $)$. To explore the second possible hypothesis, we collapsed across same and different conditions, and tested whether (DifferentCond. Rewarded + SameCond. $\left._{\text {Rewarded }}\right) / 2-$

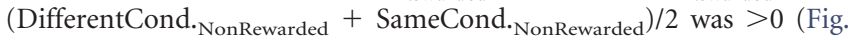
$2 d$ ). In both analyses, we corrected for multiple comparisons (number of ROIs being tested) with an FWE threshold of $p<0.05$.
Last, to investigate the relevance for behavior of the effect of motivation on representational structure, we correlated this effect with behavioral data. Specifically, for each participant, we computed the average decrease in dissimilarity and in the inverse efficiency scores (Townsend and Ashby, 1978) linked to rewarded trials (in comparison with nonrewarded ones). The inverse efficiency score was used in this analysis to take into account, simultaneously, improvements in accuracy and response speed. As we performed as many correlations as ROIs assessed in this analysis, we again controlled for multiple comparisons with an FWE threshold of $p<0.05$.

Additionally, to explore the possibility of motivation exerting an effect during the subsequent implementation of instructions, we also ran the analyses detailed above with $\beta$ images obtained from this stage.

Multivoxel pattern analysis (MVPA)based assessment of reward effects

Finally, to further connect our results with previous findings, we performed MVPA to explore the effect of reward on decoding precisions (Etzel et al., 2016). We decoded the two conditions of each of our three control-related variables, training three binary classifiers: one for distinguishing between within versus across-dimension integration instructions, other for single versus sequential response requirements, and the last one for faces and foodrelated trials. This was done separately for rewarded and nonrewarded trials. Again, we used non-normalized and unsmoothed trialwise $\beta$ images from the encoding stage. As we aimed to detect any region with reward-related increases in task decodability, we performed the MVPA in a whole-brain fashion, using searchlight (instead of biasing the results using ROIs resulting from the RSA). In each searchlight iteration, we followed a leave one-run-out cross-validation approach, training a linear support-vector machine classifier $(\mathrm{C}=1)$ (Pereira et al., 2009) with five of our six runs, and testing it with the remaining one, in an iterative fashion. Then, for each of our variables, we subtracted the accuracy map obtained from nonrewarded trials to the map from rewarded ones, and then normalized and smoothed these images, to conduct an above zero onesample $t$ test at the group level. This way, we assessed the benefits in classification precision associated with reward.

\section{Results}

\section{Behavioral results}

We analyzed RT and accuracy data separately, conducting two repeated-measures ANOVAs with four factors, corresponding to the four variables manipulated: dimension integration (within vs across), response set complexity (single vs sequential), category (faces vs food items), and motivation (rewarded vs nonrewarded). Importantly, the main effect of motivation was statistically significant on both accuracy $\left(F_{(1,31)}=4.97, p<0.05, \eta_{\mathrm{p}}^{2}=\right.$ $0.14)$ and $\operatorname{RT}\left(F_{(1,31)}=6.52, p<0.05, \eta_{\mathrm{p}}^{2}=0.17\right)$ data, with more accurate (rewarded: $\mathrm{M}=0.85, \mathrm{SD}=0.11$; nonrewarded: $\mathrm{M}=$ $0.83, \mathrm{SD}=0.12$ ) and faster (rewarded: $\mathrm{M}=1.16, \mathrm{SD}=0.21$; nonrewarded: $\mathrm{M}=1.20, \mathrm{SD}=0.20)$ responses on the rewarded condition (Fig. 3). This indicates that participants made use of 
reward cues and the economic incentives had the expected effect on behavior, improving its efficiency.

In addition, accuracy data showed a main effect of dimension integration $\left(F_{(1,31)}=9.24, p<0.05, \eta_{p}^{2}=0.23\right)$, with better performance when within-dimension integration was required (within dimension: $\mathrm{M}=0.86, \mathrm{SD}=0.13$; across dimensions: $\mathrm{M}=0.83, \mathrm{SD}=$ 0.12 ), and a significant three-way interaction of category, response set complexity, and dimension integration $\left(F_{(1,31)}=4.46, p=0.043\right.$, $\left.\eta_{\mathrm{p}}^{2}=0.13\right)$. Even despite the lack of hypothesis regarding an interaction at this level, we performed post hoc pairwise comparisons, which revealed that the interaction was driven by less robust $(p>0.05)$ differences among within and across-dimensions trials that required a single response and was food-related (while, in the rest of combinations of independent variables, this difference was significant).

On the other hand, RT results also showed a main effect of dimension integration $\left(F_{(1,31)}=61.81, p<0.001, \eta_{\mathrm{p}}^{2}=0.67\right)$ in the same direction as above (within-dimension: $\mathrm{M}=1.12, \mathrm{SD}=$ 0.17 ; across-dimensions: $\mathrm{M}=1.24, \mathrm{SD}=0.2$ ), and a main effect of category $\left(F_{(1,31)}=74.89, p<0.001, \eta_{\mathrm{p}}^{2}=0.71\right)$, with faster responses to food-related instructions (faces: $\mathrm{M}=1.23, \mathrm{SD}=$ 0.21 ; food items: $M=1.14, S D=0.19)$. Neither the effect of response set complexity (accuracy: $F_{(1,31)}=0.31, p=0.579, \eta_{\mathrm{p}}^{2}=$ 0.01 ; RT: $\left.F_{(1,31)}=0.21, p=0.653, \eta_{\mathrm{p}}^{2}=0.01\right)$ nor any other ANOVA term resulted in significant behavioral measures (main effect of category on accuracy: $F_{(1,31)}=3.23, p=0.082, \eta_{\mathrm{p}}^{2}=$ 0.094; all interactions terms, except the ones stated above, $p>$ $0.1)$.

\section{Univariate results: reward-related activations during instruction encoding}

We first assessed mean activity during novel instruction encoding, comparing rewarded against nonrewarded trials. To do so, we performed a univariate GLM, defining regressors for each combination of variables (e.g., within-dimension integration, single response, face-related rewarded trials), separately for the encoding and the implementation stages. A group-level $t$ test showed that, in accordance with our expectations and previous literature (Parro et al., 2018), the basal ganglia and FP cortices were more active for rewarded than nonrewarded instruction encoding. We observed peaks of activation (Fig. 4) in the bilateral inferior frontal junction (IFJ), premotor and supplementary motor areas (left: $[-33,5,26], z=5.07, k=489$; right: [33, 2, 59], $z=4.79, k=572)$, cingulate cortex $([-9,5,32], z=5.48, k=$ 20 ), bilateral IPS extending into the precuneus (left: $[-18,-64$, $35], z=4.77, k=357$; right: [33, $-52,53], z=4.36, k=324)$, the accumbens, ventral portion of the caudate and thalamus ([12, $-22,20], z=5.13, k=1176)$, inferior temporal gyrus $([48,-58$, $-13], z=4.48, k=52)$, occipital cortex $([30,-61,-25], z=5$, $k=1364)$, and midbrain $([0,-31,-4], z=5.19, k=255)$. Thus, regions involved in reward processing (Haber and Knutson, 2010), as well as in cognitive control paradigms with monetary incentive manipulations (e.g., Engelmann, 2009), were engaged by our task, indicating the success of the reward manipulation.

Model-based RSA results: instruction encoding structured by proactive-control variables

We aimed to identify regions whose organization during task encoding was explained by dimension integration, response set complexity, and target category. With that purpose, we used an RSA (Kriegeskorte et al., 2008) to compare the RDMs found in neural data during the encoding stage with theoretical RDMs corresponding to the three proactive control-related variables (Fig. 2). In neural RDMs, each cell contained the dissimilarity

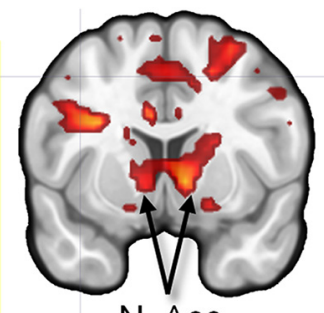

N. Acc

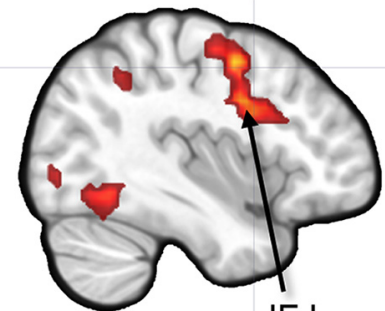

IFJ
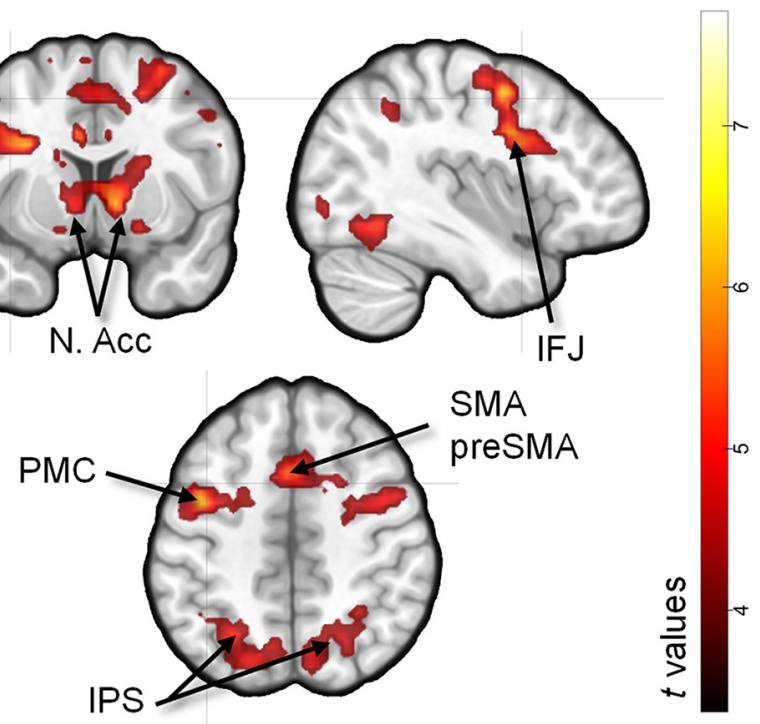

Figure 4. Regions showing greater activity during the encoding of rewarded compared with nonrewarded instructions. N. Acc, Nucleus accumbens; PMC, premotor cortex.

( 1 = Pearson correlation) between the multivariate patterns of activation of two trials. In the theoretical RDMs, cells contained dissimilarities $(1=$ maximal; $0=$ minimal $)$ that we would expect if a certain variable organized encoding (i.e.: for target category, all faces-related trials would be minimally dissimilar, while face and food-related trials would be maximally dissimilar). Using searchlight (Kriegeskorte et al., 2006), we Spearman-correlated neural and theoretical RDMs across the brain and obtained maps showing how well these three variables captured the representational space of different areas. The modality of dimension integration (Fig. $5 a$ ) only had a significant effect on rule encoding at the left MFG and IFG, incurring into the IFS $([-51,20,26], k=$ 642). Response set complexity (Fig. 5b), on the other hand, organized task representations on a wide cluster, including the bilateral IFG, premotor, supplementary, and primary motor cortices, somatosensory area, middle temporal gyrus, and superior and inferior parietal lobe extending along the IPS $([-42,-31,44]$, $k=8583)$ and in the left parahippocampal cortex $([-18,-40,-1]$, $k=301$ ). Finally, in the case of the target category RSA (Fig. 5c), significant correlations were found in an extensive cluster on the left hemisphere covering the IFG incurring into the IFJ, the fusiform gyrus, the temporoparietal junction, the inferior and middle temporal gyrus and the precuneus $([-39,-67,17], k=5581)$. On the right hemisphere, the analysis was also significant at the right middle temporal gyrus and temporoparietal junction $([39,-58,23], k=442)$ and the IFG $([42,26,14], k=295)$. Finally, the medial superior frontal gyrus $([-9,53,26], k=377)$ was also involved.

As instructions' length and speed of responses varied among some of our variables, we performed an additional multiple regression analysis, in which we included our three theoretical models, an RDM based on dissimilarities in length, and another one based on RT as regressors. Importantly, the multiple regression statistical model was examined to detect an excess of collinearity, which could have impaired the interpretability of these results. We computed the variance inflation factor for all the regressors and across our whole sample of participants, and all were $<1.1$, an index of good estimability of regression weights. The $\beta$ maps (one per model) obtained after iterating the analysis in a searchlight procedure ensured that the variance linked to our RSA models was not misattrib- 

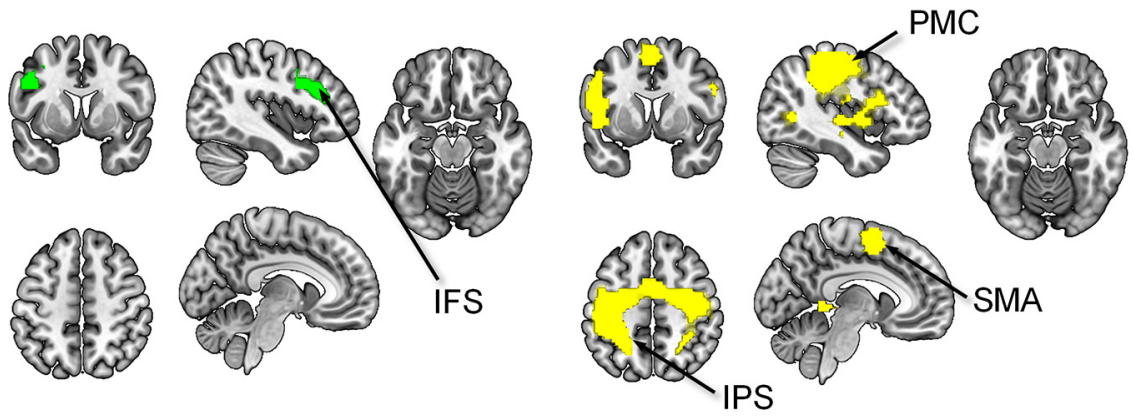

a Task complexity model

\section{b Response complexity model}
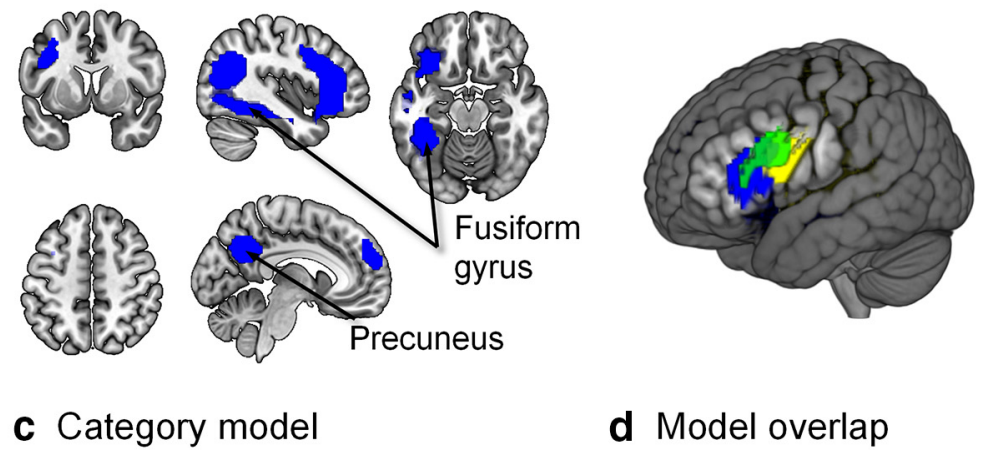

d Model overlap

Figure 5. Model-based RSA searchlight results for the three models $(\boldsymbol{a}-\boldsymbol{c})$ and render image showing the overlap among them $(\boldsymbol{d})$. Identical sections were used to display the results across models.

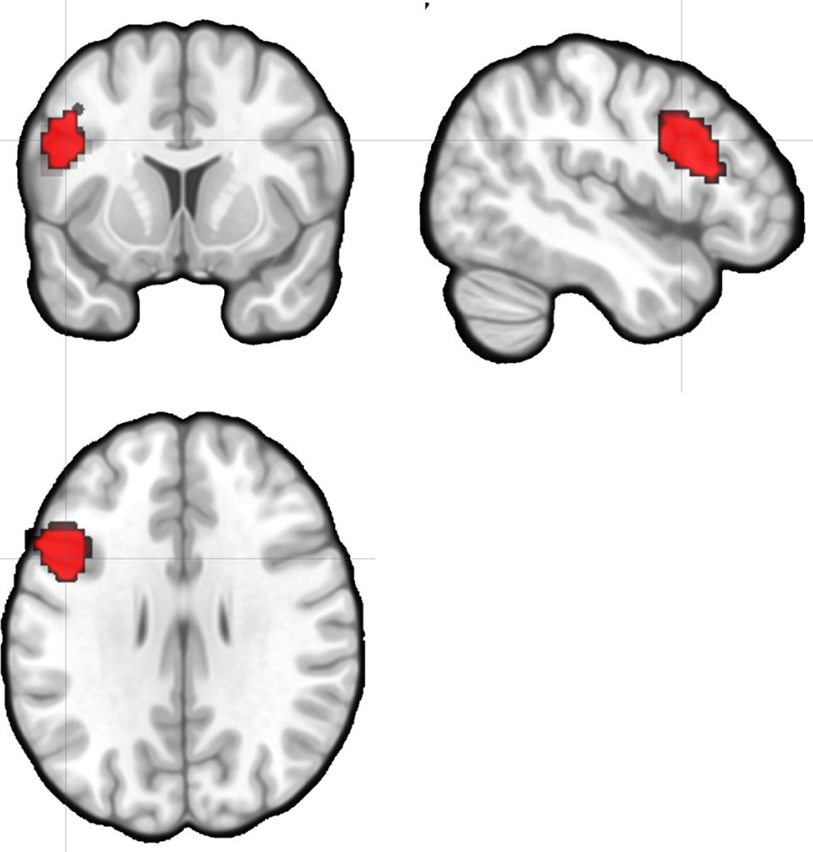

Figure 6. Conjunction analysis results.

uted due to differences in instruction length or speed of responses. Importantly, the results obtained this way were very similar to the ones extracted with the standard approach, identifying the same clusters as before.

We also conducted a conjunction analysis to assess the overlap among regions common to the three organizational schemes. Only the left IFG and IFJ resulted significant in this test (Fig. 6).

\section{LOSO-based ROI analysis: assessing confluence of models} within regions

The previous analyses left unexplained the extent to which each of the brain areas isolated by RDM analyses reflected in their organization the three manipulated variables. Furthermore, the conservative correction for multiple comparisons used in the searchlight could overshadow this effect elsewhere in the brain. To shed some light upon this issue, we used a more sensitive ROI analysis, together with an LOSO approach to avoid double dipping when selecting regions.

All the clusters identified in the main group results (Fig. 5) were consistently found across all participants with the LOSO approach, with the exception of the medial superior frontal gyrus under the category model, which was absent in four subjects and thus not included in the analysis. The correlations of the ROIs' RDMs and the three models' matrices were analyzed with a repeated-measures ANOVA, in which we found a significant interaction of ROI and Model $\left(F_{(12,348)}=6.050, p<0.001, \eta_{\mathrm{p}}^{2}=\right.$ 0.173 ), evidencing variability in instruction coding structure across regions. We then ran one-sample $t$ tests or Wilcoxon signed-rank tests (depending on data distribution) to assess model performance in each ROI (Table 1). The general pattern obtained replicated the searchlight results: the model that originally identified each specific ROI in the searchlight was the one explaining most robustly its encoding activity. Further, in almost all the regions, we did not find enough evidence supporting the effect of the remaining variables. Converging with the previous analyses, the left IFG identified with the dimension integration model was also significantly correlated with response set complexity and category. Similarly, the left IFG cluster found in the category RSA was correlated with the dimension integration model too. In addition, this confluence of models analysis revealed that the response set model was also significant in the 
Table 1. Effect of the three models on the LOSO-estimated ROIs ${ }^{a}$

\begin{tabular}{|c|c|c|c|c|c|}
\hline Original model & ROI & $\begin{array}{l}\text { Model } \\
\text { tested }\end{array}$ & $t$ & $Z$ & $p$ \\
\hline \multirow{3}{*}{$\begin{array}{l}\text { Dimension } \\
\text { integration }\end{array}$} & \multirow[t]{3}{*}{ Left IFG } & Dim. & 3.354 & & 0.0 \\
\hline & & Resp. & 3.292 & & 0.00 \\
\hline & & Cat. & 3.635 & & 0.0 \\
\hline \multirow{6}{*}{$\begin{array}{l}\text { Response set } \\
\text { complexity }\end{array}$} & \multirow[t]{3}{*}{ Left IPS } & Dim. & 0.614 & & 1 \\
\hline & & Resp. & 5.351 & & $<0.00$ \\
\hline & & Cat. & & 1.975 & 0.16 \\
\hline & \multirow[t]{3}{*}{ Motor cortices, left LPFC } & Dim. & 2.478 & & 0.06 \\
\hline & & Resp. & 3.647 & & 0.00 \\
\hline & & Cat. & 1.166 & & 0.88 \\
\hline \multirow[t]{12}{*}{ Target category } & \multirow{3}{*}{$\begin{array}{l}\text { Left fusiform gyrus } \\
\text { and precuneus }\end{array}$} & Dim. & 0.476 & & 1 \\
\hline & & Resp. & 3.463 & & 0.00 \\
\hline & & Cat. & 5.466 & & $<0.00$ \\
\hline & \multirow[t]{3}{*}{ Left IFG } & Dim. & 2.832 & & 0.02 \\
\hline & & Resp. & & 0.699 & 0.24 \\
\hline & & Cat. & 4.930 & & $<0.00$ \\
\hline & \multirow[t]{3}{*}{ Right MTG } & Dim. & & -0.144 & 0.55 \\
\hline & & Resp. & & -1.008 & 0.84 \\
\hline & & Cat. & & 2.859 & 0.00 \\
\hline & \multirow[t]{3}{*}{ Right IFG } & Dim. & & 1.275 & 0.10 \\
\hline & & Resp. & & -0.206 & 0.58 \\
\hline & & Cat. & & 3.085 & 0.00 \\
\hline
\end{tabular}

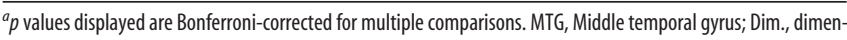
sion integration model; Resp., response complexity model; Cat., target category.

category-related cluster involving the left fusiform and precuneus (Table 1).

ROI analysis spanning multiple demand network regions

Following a similar strategy as above, we also examined task encoding organization across the regions comprising the MD network. We extracted each MD region's RDM and correlated it with our three models' RDM, and then entered the correlation coefficients into a repeated-measures ANOVA. Again, a significant ROI $\times$ Model interaction was found $\left(F_{(20,620)}=2.168, p=\right.$ $\left.0.002, \eta_{\mathrm{p}}^{2}=0.065\right)$. To assess which models significantly structured activations across MD ROIs, we conducted one-sample $t$ tests or Wilcoxon signed-rank tests when data were not normally distributed (Table 2).

Only a subset of MD network regions encoded instructions consistently according to any of the proactive control variables, and all of them were located on the left hemisphere and in the LPFC and parietal cortex. The findings were, however, consistent with the searchlight and ROI-related results presented so far. The three variables exerted an effect on different left lateral prefrontal sections: dimension integration and response complexity on the IFG; dimension integration and target category on the more dorsal MFG; and finally, category on the RLPFC. Response complexity was the attribute that most robustly captured representational organization in the IPS.

\section{Effects of reward on representational geometry}

We then explored the effects of motivation in each of the ROIs encoding different attributes of the instructions (Fig. 5), assessing two possible mechanisms that could underlie the behavioral improvements linked to reward (Fig. 2). On the one hand, we tested whether reward made our variables more efficient in sharpening the representational space (Fig. $2 d$, Hypothesis 1). In other words, and taking as an example the target category variable, we assessed whether reward expectations increased the distance between representations of instructions referring to different stimulus categories (in extension to the other variables, indicated as
Table 2. Effect of the three models on the MD network ROIs ${ }^{a}$

\begin{tabular}{|c|c|c|c|c|}
\hline ROI & $\begin{array}{l}\text { Model } \\
\text { tested }\end{array}$ & $t$ & $z$ & $p$ \\
\hline \multirow[t]{3}{*}{ ACC/pre-SMA } & Dim. & & 0.645 & 1 \\
\hline & Resp. & & 1.673 & 0.115 \\
\hline & Cat. & -0.026 & & 1 \\
\hline \multirow[t]{3}{*}{ Left RLPFC } & Dim. & & 1.019 & 0.571 \\
\hline & Resp. & & 0.346 & 0.365 \\
\hline & Cat. & & 2.665 & 0.023 \\
\hline \multirow[t]{3}{*}{ Left IFS } & Dim. & 3.644 & & 0.005 \\
\hline & Resp. & 4.423 & & $<0.001$ \\
\hline & Cat. & & 2.328 & 0.058 \\
\hline \multirow[t]{3}{*}{ Left MFG } & Dim. & & 2.739 & 0.014 \\
\hline & Resp. & & 0.870 & 0.754 \\
\hline & Cat. & 4.298 & & 0.002 \\
\hline \multirow[t]{3}{*}{ Left alfo } & Dim. & 0.667 & & 1 \\
\hline & Resp. & & 1.206 & 0.228 \\
\hline & Cat. & & 2.197 & 0.060 \\
\hline \multirow[t]{3}{*}{ Left IPS } & Dim. & 1.617 & & 0.638 \\
\hline & Resp. & & 2.814 & 0.025 \\
\hline & Cat. & 2.639 & & 0.071 \\
\hline \multirow[t]{3}{*}{ Right RLPFC } & Dim. & & 0.365 & 1 \\
\hline & Resp. & 1.460 & & 0.849 \\
\hline & Cat. & 0.861 & & 1 \\
\hline \multirow[t]{3}{*}{ Right IFS } & Dim. & 2.220 & & 0.186 \\
\hline & Resp. & & 1.599 & 0.211 \\
\hline & Cat. & & -0.626 & 1 \\
\hline \multirow[t]{3}{*}{ Right MFG } & Dim. & 2.311 & & 0.152 \\
\hline & Resp. & 1.294 & & 1 \\
\hline & Cat. & 2.042 & & 0.273 \\
\hline \multirow[t]{3}{*}{ Right alf0 } & Dim. & 0.023 & & 1 \\
\hline & Resp. & & 1.299 & 0.280 \\
\hline & Cat. & 1.352 & & 1 \\
\hline \multirow[t]{3}{*}{ Right IPS } & Dim. & & 1.262 & 0.548 \\
\hline & Resp. & & 1.842 & 0.330 \\
\hline & Cat. & & -0.701 & 1 \\
\hline
\end{tabular}

${ }^{a} p$ values displayed are Bonferroni-corrected for multiple comparisons. Dim., Dimension integration model; Resp., response complexity model; Cat., target category.

different-condition dissimilarity), while decreasing the distance among those referring to same target category (same-condition dissimilarity). On the other, we tested the alternative possibility that dissimilarities would be, in general, greater in the rewarded trials (Fig. 2d, Hypothesis 2), regardless of the variables manipulated (i.e., regardless of the pair of instructions being same or different-condition). This could reflect a mechanism for making rule representations more distinguishable among each other; and also, it would be compatible with the increase in rule decoding accuracy that has been liked to motivation in previous reports (Etzel et al., 2016). With that purpose, we extracted, for each region, the average dissimilarity among pairs of instructions pertaining to the same and different conditions, separately for rewarded and nonrewarded trials. We then used Wilcoxon signed-rank tests (Nili et al., 2014) to check whether the difference between different-condition and same-condition trials was larger in the rewarded than in the nonrewarded condition, and also, whether the mean dissimilarity (collapsing across same and different-condition) was increased by motivation.

In the first case, no reward-related differences were observed for any of the instruction-related variables (all $p$ values $>0.1$ ). It is important to note, however, that these results (as most of the findings presented in this study) are anchored to the instruction's encoding stage, in which proactive configuration takes place. To explore the possibility that the hypothesized interaction shaped neural activations during the later implementation phase (more 


\begin{tabular}{|c|c|c|}
\hline ROI & $\begin{array}{l}\text { Effect of reward on } \\
\text { dissimilarity values }\end{array}$ & $\begin{array}{l}\text { Correlation RSA } \\
\text { and behavior }\end{array}$ \\
\hline \multicolumn{3}{|l|}{ Task set complexity } \\
\hline Left IFG/IFJ & $z=-3.005^{*}$ & $r=0.515^{*}$ \\
\hline \multicolumn{3}{|l|}{ Response set complexity } \\
\hline M1/PM/SMA/IPS & $z=-3.712^{*}$ & $r=0.565^{*}$ \\
\hline Left PHC & $z=-3.712^{*}$ & $r=0.558^{*}$ \\
\hline \multicolumn{3}{|l|}{ Target category } \\
\hline Left fusiform gyrus/precuneus/IFG/IFJ & $z=-3.712^{*}$ & $r=0.543^{*}$ \\
\hline Right MTG/TPJ & $z=-4.419^{*}$ & $r=0.495^{*}$ \\
\hline Right IFG & $z=-3.712^{*}$ & $r=0.533^{*}$ \\
\hline Medial SFG & $z=-2.652^{*}$ & $r=0.482^{*}$ \\
\hline
\end{tabular}

${ }^{a}$ In the last case, multiple comparisons were controlled with an FWE criterion. M1, Primary motor cortex; PM, premotor cortex; PHC, parahippocampal cortex; MTG, middle temporal gyrus; TPJ, temporoparietal junction; SFG, superior frontal gyrus.

${ }^{*} p<0.05$, Wilcoxon paired-sample signed-rank test (middle column) or Pearson correlation coefficient (left column).

related to reactive control; Braver, 2012; Palenciano et al., 2019), we conducted a further test using $\beta$ images from this epoch. However, and again, the expected effect was not significant for any of the ROIs examined (all $p$ values $>0.1$ ).

When addressing the second hypothesis, surprisingly, we found the opposite pattern: reward systematically decreased the dissimilarity values in all the ROIs evaluated (all $p$ values $<0.05$; Table 3 ). To test the behavioral relevance of this finding we correlated, across our participants, the average decrease in dissimilarities associated with reward, with the benefit of motivation on performance (inverse efficiency score; Townsend and Ashby, 1978). We found that, indeed, the decrease in representational distances due to reward was significantly correlated with the motivation-related improvements in behavioral performance. Furthermore, this seemed to be a quite robust effect, being present in all of the ROIs included in the analysis (for further details, see Table 3).

\section{MVPA results}

We finally aimed to explore the effect of reward directly on decoding accuracies, using MVPA (Haxby et al., 2014), as it has been previously reported during rule encoding in a classic, repetitive task-switching setting (Etzel et al., 2016). We discriminated between the two conditions of each instruction-related variable (i.e., one among faces and food-related trials, other for single vs sequential response requirements, and a last one for within vs across-dimension integration instructions) separately for rewarded and nonrewarded trials. We trained and tested our classifiers across the whole brain using searchlight and obtained, as a result, an accuracy map for each motivation condition and variable. Nonetheless, while classification was above chance in different brain regions for the three variables, we did not detect any differences in accuracies between rewarded and nonrewarded trials, as no cluster survived at the group level the $t$ test assessing above 0 differences between the two motivation conditions.

\section{Discussion}

In the present study, we aimed to characterize the representational space for novel instructions during their proactive preparation. We assessed whether variables linked to proactive control organized encoding activity patterns and whether this structure was affected by reward expectations. Our results portrayed a complex landscape, where different organizational principles governed instruction encoding in FP cortices and lower-level perceptual and motor areas.
The left IFG/IFJ reflected the most complex and overarching representational structure, with activity patterns structured by dimension integration, response complexity, and target category. Robust evidence supports the role of the IFJ in task-set reconfiguration (Brass et al., 2005) in practiced (e.g., Woolgar et al., 2011) and novel contexts (e.g., González-García et al., 2016; MuhleKarbe et al., 2017), orchestrating neural dynamics during attentional selection (e.g., Baldauf and Desimone, 2014). This region seems to be involved in task-set maintenance (Sakai, 2008), selecting task-relevant information represented in perceptual regions (Miller and Cohen, 2001; Cole et al., 2013). The current study advances our knowledge about the structure underlying how information is coded during novel instruction encoding, and stresses the diversity of task parameters that orchestrate task encoding in the IFG/IFJ. Such a complex, multidimensional representational space (Rigotti et al., 2013) could be key to support the richness and flexibility of human behavior in novel environments. This perspective qualifies recent research, based on MVPA, that highlights the compositionality characterizing representations held in the IFG (Reverberi et al., 2012; Cole et al., 2013; Deraeve et al., 2019), by which complex tasks are coded by combining their simpler constituent elements.

The IPS also encoded novel rules proactively, but now according to response complexity. While this is consistent with previous studies linking the parietal cortex to action preparation, it is worth noticing the distinction found in our data between parietal and prefrontal regions, a finding further confirmed with a more sensitive ROI analysis. Dimension integration, the variable manipulated to appeal to a higher-level task goal representation, had an effect only on LPFC, while the IPS was linked to the more specific response-set complexity (Rubinstein et al., 2001; De Baene and Brass, 2014). The frequent coactivation of IFG/IFJ and IPS in demanding paradigms (Duncan, 2010) had complicated the identification of their separate contributions. The differential pattern we observed is highly relevant to disentangle their proactive role. Interestingly, the emerging picture portraits the IFG/IFJ and the IPS collaborating during novel task representation, with the former maintaining overarching representations of all relevant variables, and the latter activating the relevant stimulus-response contingencies (see also Muhle-Karbe et al., 2014). The use of RSA in our paradigm provides a deeper understanding of this process, emphasizing that the proposed two-stage preparatory mechanism also guides task-set encoding in FP cortices. In this sense, variables key for abstract goals or specific stimulus-response mappings become relevant differentially depending on the region.

Additional medial and lateral frontal cortices also participate in the FP network and are frequently recruited during task preparation (Duncan, 2010). Consequently, we also examined instruction coding in these MD regions. Our findings highlighted other LPFC areas reflecting target category (both the RLPFC and MFG) and dimension integration (MFG). The overall pattern of results obtained both with whole-brain and with ROI approaches reflects high heterogeneity within the FP network in general, and in the LPFC in particular, in terms of the attributes structuring task-set representation. In contrast, we did not obtain evidence supporting proactive task-set encoding in the ACC/pre-SMA and the aIfO regions. This finding fits with the subdivision of the FP network into two differentiated components: one anchored in the LPFC and IPS, and a second one composed by the ACC and the aIfO (Dosenbach et al., 2007; Palenciano et al., 2019). In line with our results, anticipatory task coding has been predominantly found in regions from the former rather than in the latter (Crittenden et al., 2016). Ultimately, the variability found within the FP 
control network during proactive novel task setting (Palenciano et al., 2019), with different processes and representational formats being combined, could be key to maximize flexibility.

FP cortices were not the sole brain regions encoding novel instruction parameters. Activity in fusiform gyri was organized according to target category, whereas patterns in somatomotor cortices reflected response complexity. While these regions are not associated per se with proactive control, their involvement reflects that their representational geometry is tuned in an anticipatory fashion by relevant task parameters conveyed by instructions. It is important to stress that all the results discussed were locked to instruction encoding, where no target stimuli had been presented, neither any specific motor response could have been prepared. These findings suggest that FP areas exert a bias in posterior cortices, according to the content of instructions. Supporting this, increments of mean activity (Esterman and Yantis, 2010) and target-specific information encoding (e.g., Stokes et al., 2009) have been reported in perceptual and motor regions during preparation. Importantly, these changes have been linked to boosts in functional connectivity between the FP and posterior cortices (Sakai and Passingham, 2006; González-García et al., 2016). In direct relation to our findings, a recent study showed that the representational organization in regions along the visual pathway is dynamically adapted to task demands (Nastase et al., 2017). Our current results add to these findings by showing that representational space tuning could be a mechanism of preparatory bias, which could reflect predictive coding principles where iterative loops of feedback and feedforward communication shape cognition (Friston, 2005).

Crucially, the structure of information encoded by all these regions was sensitive to trialwise motivational states. Surprisingly, reward expectation diminished the dissimilarities between the representations of the instructions, yet preserving the organizational scheme found in each area. Based on recent findings of increased task decodability (Etzel et al., 2016), we had hypothesized that reward would either polarize the representational structure or increase the representational distances overall. Results were, however, in the opposite direction, even when our reward manipulation was successful at boosting performance and also increased activity in control and reward-related regions (Parro et al., 2018). Most importantly, decreases in dissimilarities were also robustly correlated with behavioral improvements. Taking into account that additional analysis using MVPA and using data from the implementation stage corroborated these results, their implication must be thoughtfully considered. One possibility is that the decrease in dissimilarities is generated by a general boost of reward in signal-to-noise ratio. Although our results persisted after normalizing data across trials, a rewardrelated reduction of multivariate noise pattern could still be possible, and it could benefit task coding in the absence of the hypothesized RSA results. However, the MVPA did not reveal improved task classification accuracy in the rewarded condition; thus, this interpretation remains uncertain. Alternatively, motivation could have influenced task coding in ways that our searchlight procedure was not sensitive to. That would be the case if reward affected the spatial distribution of information: as ROIs were defined by size-fixed searchlight spheres, and were equal in rewarded and nonrewarded conditions, an effect like that would remain shadowed. Finally, task complexity could also be key. In less demanding situations, such as repetitive task switching (Etzel et al., 2016), reward could directly sharpen task encoding representations. In novel environments, however, motivation could exert a more general effect at the process level instead of at the representational one. It could increase the efficiency of task reconfiguration (Braem and Egner, 2018), as indexed by the improvements in behavior, while the specific rule representations would remain equally structured. Nonetheless, more research is needed to properly characterize the intricate interactions among proactive control and motivation (Pessoa, 2017) in rich task environments, more akin to daily life situations.

The current study entails some limitations that constrain the scope of our findings and call for further research. On the one hand, the nature of our paradigm demanded the selection of a few instruction-organizing variables. Some other dimensions, critical for anticipatory encoding, may have been left unaddressed. Furthermore, nonlinear combinations of variables could add to the organization principles governing control regions (Rigotti et al., 2013). Considering an increasing number of plausible models in more complex and/or naturalistic scenarios, together with datadriven methods, such as multidimensional scaling or component analysis, will complement our results. On the other hand, our main dependent variable (fMRI hemodynamic signal) provided spatially precise, but temporally impoverished data. Temporally resolved techniques, such as EEG or MEG, could be key to unveil the temporal dynamics of the representational patterns.

Overall, our findings provide novel insights on how verbal complex novel instructions organize proactive brain activations. The emerging picture departs from pure localizationist approaches where brain regions carry fixed information about concrete cognitive processes. Rather, the different dimensions relevant for efficient instructed action shape brain activity across an extended set of areas, flexibly structuring encoding activity according to the relevant task parameters.

\section{References}

Arco JE, González-García C, Díaz-Gutiérrez P, Ramírez J, Ruz M (2018) Influence of activation pattern estimates and statistical significance tests in fMRI decoding analysis. J Neurosci Methods 308:248-260.

Baldauf D, Desimone R (2014) Neural mechanisms of object-based attention. Science 344:424-427.

Bourguignon NJ, Braem S, Hartstra E, De Houwer J, Brass M (2018) Encoding of novel verbal instructions for prospective action in the lateral prefrontal cortex: evidence from univariate and multivariate functional magnetic resonance imaging analysis. J Cogn Neurosci 30:1170-1184.

Braem S, Egner T (2018) Getting a grip on cognitive flexibility. Curr Dir Psychol Sci 27:470-476.

Brass M, Liefooghe B, Braem S, De Houwer J (2017) Following new task instructions: evidence for a dissociation between knowing and doing. Neurosci Biobehav Rev 81:16-28.

Brass M, Derrfuss J, Forstmann B, von Cramon DY (2005) The role of the inferior frontal junction area in cognitive control. Trends Cogn Sci 9:314316.

Braver TS (2012) The variable nature of cognitive control: a dual mechanisms framework. Trends Cogn Sci 16:106-113.

Brett M, Anton J, Valabregue R, Poline J (2002) Region of interest analysis using the MarsBar toolbox for SPM 99. Neuroimage 16:99.

Cole MW, Bagic A, Kass R, Schneider W (2010) Prefrontal dynamics underlying rapid instructed task learning reverse with practice. J Neurosci 30: 14245-14254

Cole MW, Laurent P, Stocco A (2013) Rapid instructed task learning: a new window into the human brain's unique capacity for flexible cognitive control. Cogn Affect Behav Neurosci 13:1-22.

Cole MW, Reynolds JR, Power JD, Repovs G, Anticevic A, Braver TS (2013) Multi-task connectivity reveals flexible hubs for adaptive task control. Nat Neurosci 16:1348-1355.

Cole MW, Ito T, Braver TS (2016) The behavioral relevance of task information in human prefrontal cortex. Cereb Cortex 26:2497-2505.

Cole MW, Patrick LM, Braver TS (2018) A role for proactive control in rapid instructed task learning. Acta Psychol 184:20-30.

Cole MW, Braver TS, Meiran N (2017) The task novelty paradox: flexible 
control of inflexible neural pathways during rapid instructed task learning. Neurosci Biobehav Rev 81:4-15.

Connolly AC, Guntupalli JS, Gors J, Hanke M, Halchenko YO, Wu YC, Abdi H, Haxby JV (2012) The representation of biological classes in the human brain. J Nuerosci 32:2608-2618.

Crittenden BM, Mitchell DJ, Duncan J (2016) Task encoding across the multiple demand cortex is consistent with a frontoparietal and cinguloopercular dual networks distinction. J Neurosci 36:6147-6155.

De Baene W, Brass M (2014) Dissociating strategy-dependent and independent components in task preparation. Neuropsychologia 62:331-340.

Deraeve J, Vassena E, Alexander W (2019) Conjunction or co-activation? A multi-level MVPA approach to task set representations. BioRxiv 521385.

Dosenbach NU, Fair DA, Miezin FM, Cohen AL, Wenger KK, Dosenbach RA, Fox MD, Snyder AZ, Vincent JL, Raichle ME, Schlaggar BL,Petersen SE (2007) Distinct brain networks for adaptive and stable task control in humans. Proc Natl Acad Sci U S A 104:11073-11078.

Dumontheil I, Thompson R, Duncan J (2011) Assembly and use of new task rules in fronto-parietal cortex. J Cogn Neurosci 23:168-182.

Duncan J (2010) The multiple-demand (MD) system of the primate brain: mental programs for intelligent behaviour. Trends Cogn Sci 14:172-179.

Duncan J, Parr A, Woolgar A, Thompson R, Bright P, Cox S, Bishop S,Nimmo-Smith I (2008) Goal neglect and Spearman's g: competing parts of a complex task. J Exp Psychol Gen 137:131-148.

Engelmann JB, Damaraju E, Padmala S, Pessoa L (2009) Combined effects of attention and motivation on visual task performance: transient and sustained motivational effects. Front Hum Neurosci 3:4.

Esterman M, Yantis S (2010) Perceptual expectation evokes categoryselective cortical activity. Cereb Cortex 20:1245-1253.

Esterman M, Tamber-Rosenau BJ, Chiu YC, Yantis S (2010) Avoiding nonindependence in fMRI data analysis: leave one subject out. Neuroimage 50:572-576.

Etzel JA, Cole MW, Zacks JM, Kay KN, Braver TS (2016) Reward motivation enhances task coding in frontoparietal cortex. Cereb Cortex 26:1647-1659.

Fedorenko E, Duncan J, Kanwisher N (2013) Broad domain generality in focal regions of frontal and parietal cortex. Proc Natl Acad Sci U S A 110:16616-16621.

Friston K (2005) A theory of cortical responses. Philos Trans R Soc Lond B Biol Sci 360:815-836.

González-García C, Mas-Herrero E, de Diego-Balaguer R, Ruz M (2016) Task-specific preparatory neural activations in low-interference contexts. Brain Struct Funct 221:3997-4006.

González-García C, Arco JE, Palenciano AF, Ramírez J, Ruz M (2017) Encoding, preparation and implementation of novel complex verbal instructions. Neuroimage 148:264-273.

Haber SN, Knutson B (2010) The reward circuit: linking primate anatomy and human imaging. Neuropsychopharmacology 35:4-26.

Hartstra E, Kühn S, Verguts T, Brass M (2011) The implementation of verbal instructions: an fMRI study. Hum Brain Mapp 32:1811-1824.

Haxby JV, Connolly AC, Guntupalli JS (2014) Decoding neural representational spaces using multivariate pattern analysis. Annu Rev Neurosci $37: 435-456$

Hebart MN, Görgen K, Haynes JD (2014) The Decoding Toolbox (TDT): a versatile software package for multivariate analyses of functional imaging data. Front Neuroinform 8:88.

Kriegeskorte N, Goebel R, Bandettini P (2006) Information-based functional brain mapping. Proc Natl Acad Sci U S A 103:3863-3868.

Kriegeskorte N, Mur M, Bandettini P (2008) Representational similarity analysis: connecting the branches of systems neuroscience. Front Syst Neurosci 2:4.

Liefooghe B, Wenke D, De Houwer J (2012) Instruction-based task-rule congruency effects. J Exp Psychol Learn Mem Cogn 38:1325-1335.

Luria, A. R (1966) Higher cortical functions in man. New York: Springer.

Miller EK, Cohen JD (2001) An integrative theory of prefrontal cortex function. Annu Rev Neurosci 24:167-202.

Muhle-Karbe PS, Andres M, Brass M (2014) Transcranial magnetic stimulation dissociates prefrontal and parietal contributions to task preparation. J Neurosci 34:12481-12489.

Muhle-Karbe PS, Duncan J, De Baene W, Mitchell DJ, Brass M (2017) Neural coding for instruction-based task sets in human frontoparietal and visual cortex. Cereb Cortex 27:1891-1905.
Mumford JA, Poline JB, Poldrack RA (2015) Orthogonalization of regressors in fMRI models. PLoS One 10:e126255.

Nastase SA, Connolly AC, Oosterhof NN, Halchenko YO, Guntupalli JS, Visconti di Oleggio Castello M, Gors J, Gobbini MI,Haxby JV (2017) Attention selectively reshapes the geometry of distributed semantic representation. Cereb Cortex 27:4277-4291.

Nichols T, Brett M, Andersson J, Wager T, Poline JB (2005) Valid conjunction inference with the minimum statistic. Neuroimage 25:653-660.

Nili H, Wingfield C, Walther A, Su L, Marslen-Wilson W, Kriegeskorte N (2014) A toolbox for representational similarity analysis. PLoS Comput Biol 10:e1003553.

Palenciano AF, González-García C, Arco JE, Ruz M (2019) Transient and sustained control mechanisms supporting novel instructed behavior. Cereb Cortex 29:3948-3960.

Parro C, Dixon ML, Christoff K (2018) The neural basis of motivational influences on cognitive control. Hum Brain Mapp 39:5097-5111.

Pereira F, Mitchell T, Botvinick M (2009) Machine learning classifiers and fMRI: a tutorial overview. Neuroimage 45 [Suppl 1]:S199-S209.

Pessoa L (2009) How do emotion and motivation direct executive control? Trends Cogn Sci 13:160-166.

Pessoa L (2017) Cognitive-motivational interactions: beyond boxes-andarrows models of the mind-brain. Motiv Sci 3:287-303.

Popov V, Ostarek M, Tenison C (2018) Practices and pitfalls in inferring neural representations. Neuroimage 174:340-351.

Reverberi C, Görgen K, Haynes JD (2012) Distributed representations of rule identity and rule order in human frontal cortex and striatum. J Neurosci 32:17420-17430.

Reverberi C, Görgen K, Haynes JD (2012) Compositionality of rule representations in human prefrontal cortex. Cereb Cortex 22:1237-1246.

Rigotti M, Barak O, Warden MR, Wang XJ, Daw ND, Miller EK, Fusi S (2013) The importance of mixed selectivity in complex cognitive tasks. Nature 497:585-590.

Ritchie JB, Bracci S, Op de Beeck H (2017) Avoiding illusory effects in representational similarity analysis: what (not) to do with the diagonal. Neuroimage 148:197-200.

Rubinstein JS, Meyer DE, Evans JE (2001) Executive control of cognitive processes in task switching federal aviation administration. J Exp Psychol Hum Percept Perform 27:763-797.

Sakai K (2008) Task set and prefrontal cortex. Annu Rev Neurosci 31: 219-245.

Sakai K, Passingham RE (2003) Prefrontal interactions reflect future task operations. Nat Neurosci 6:75-81.

Sakai K, Passingham RE (2006) Prefrontal set activity predicts rule-specific neural processing during subsequent cognitive performance. J Neurosci 26:1211-1218.

Stelzer J, Chen Y, Turner R (2013) Statistical inference and multiple testing correction in classification-based multi-voxel pattern analysis (MVPA): random permutations and cluster size control. Neuroimage 65:69-82.

Stokes M, Thompson R, Nobre AC, Duncan J (2009) Shape-specific preparatory activity mediates attention to targets in human visual cortex. Proc Natl Acad Sci U S A 106:19569-19574.

Tottenham N, Tanaka JW, Leon AC, McCarry T, Nurse M, Hare TA, Marcus DJ, Westerlund A, Casey BJ, Nelson C (2009) The NimStim set of facial expressions: judgments from untrained research participants. Psychiatry Res 168:242-249.

Townsend J, Ashby G (1978) Methods of modeling capacity in simple processing systems. In: Cognitive theory (Castellan J, Restle F, eds), Vol 3, pp 200-239. Hillsdale, NJ: Erlbaum.

Treisman AM, Gelade G (1980) A feature-integration theory of attention. Cogn Psychol 12:97-136.

Turner B (2010) Comparison of methods for the use of pattern classification on rapid event-related fMRI data. In: Annual Meeting of the Society for Neuroscience. San Diego, CA.

Walther A, Nili H, Ejaz N, Alink A, Kriegeskorte N, Diedrichsen J (2016) Reliability of dissimilarity measures for multi-voxel pattern analysis. Neuroimage 137:188-200

Wisniewski D, Forstmann B, Brass M (2019) Outcome contingency selectively affects the neural coding of outcomes but not of tasks. BioRxiv 375642 .

Woolgar A, Hampshire A, Thompson R, Duncan J (2011) Adaptive coding of task-relevant information in human frontoparietal cortex. J Neurosci $31: 14592-14599$. 